\title{
Hospitalization for Invasive Pneumococcal Diseases in Young Children before Use of 13-Valent Pneumococcal Conjugate Vaccine, Suzhou, China
}

Kaile Chen, Xiyan Zhang, Yunzhen Tao, Yunzhong Wang, Jian Xue, Changpeng Liu, Shuang Feng, Yongdong Yan, Qinghui Chen, Jianmei Tian, Genming Zhao, Xuejun Shao, Tao Zhang

A 13-valent pneumococcal conjugate vaccine against invasive pneumococcal disease (IPD) was introduced in China in April 2017. We describe 105 children $<5$ years of age who were hospitalized for IPD at Soochow University Affiliated Children's Hospital in Suzhou, China, during January 2010-December 2017. We calculated the incidence of hospitalization for IPD as $14.55 / 100,000$ children in Suzhou. We identified 8 different capsular serotypes: $6 \mathrm{~B}$ (28.4\% of cases), 14 (18.9\% of cases), $19 \mathrm{~A}(18.9 \%$ of cases), $19 \mathrm{~F}$ (12.2\% of cases), $23 \mathrm{~F}(10.8 \%$ of cases), 20 ( $4.1 \%$ of cases), $9 \mathrm{~V}$ ( $4.1 \%$ of cases), and $15 \mathrm{~B} / \mathrm{C}(2.7 \%$ of cases). These results provide baseline data of IPD before the introduction of this vaccine in China, enabling researchers to better understand its effects on IPD incidence.

Streptococcus pneumoniae infections are a major $\checkmark$ cause of illness and death in infants and children worldwide, especially in developing countries (1). The World Health Organization estimates that pneumococcal diseases cause $\approx 1.6$ million deaths every year, of which $\approx 1.0$ million occur in children $<5$ years of age (2). Invasive pneumococcal diseases (IPDs) such as bacteremic pneumonia, febrile bacteremia, and meningitis often are fatal, and $\approx 25 \%-50 \%$ of survivors have serious neurological sequelae (3). IPD incidence varies among countries and populations $(4,5)$. Very young or old age, concurrent conditions,

Author affiliations: Fudan University, Shanghai, China (K. Chen, X. Zhang, C. Liu, S. Feng, G. Zhao, T. Zhang); Key Laboratory of Public Health Safety, Ministry of Education, Shanghai (K. Chen, X. Zhang, C. Liu, S. Feng, G. Zhao, T. Zhang); Soochow University Affiliated Children's Hospital, Suzhou, China (Y. Tao, Y. Wang, J. Xue, Y. Yan, Q. Chen, J. Tian, X. Shao)

DOI: https://doi.org/10.3201/eid2701.181415 malnutrition, poor healthcare, and low socioeconomic status are risk factors for IPD.

The growing resistance of $S$. pneumoniae to common antimicrobial drugs highlights the importance of vaccines in preventing pneumococcal disease $(6,7)$. In China, vaccines fall under 2 categories: category I vaccines, which guard against diseases such as hepatitis B, polio, and measles, are mandatory, and are provided by the government; and category II vaccines, which are optional and commercially available. The 7-valent pneumococcal conjugate vaccine (PCV7) was licensed as a category II vaccine in mainland China in 2008; the estimated uptake rate was $2 \%-7 \%(8,9)$. The 13 -valent pneumococcal conjugate vaccine (PCV13) was introduced in mainland China in November 2016 as a category II vaccine. To evaluate the effect of PCV13 on IPD incidence, we describe baseline epidemiologic characteristics of illness, hospitalization, and death associated with the disease. Li et al. evaluated surveillance data from 4 prefecture cities (Jinan, Yichang, Shijiazhuang, and Guigang) and reported that $S$. pneumoniae was the most common cause of bacterial meningitis in children $<5$ years of age during 2006-2009 (10). However, few data exist on the incidence of pneumococcal pneumonia, sepsis, and other manifestations of IPD in different regions of mainland China.

We describe IPD among patients $<5$ years of age who were treated at Soochow University Affiliated Children Hospital (SCH) in Suzhou, China, during 2010-2017. We used a rapid method of the World Health Organization to estimate the baseline incidence of IPD hospitalization (11) among this age group. 


\section{Methods}

\section{Study Site and Catchment Area}

The study was conducted in Suzhou, a major city with a population of $\approx 12$ million persons in the southeast area of Jiangsu Province in eastern China. Suzhou consists of 5 municipal districts (Gusu, New and High-Tech, Wuzhong, Xiangcheng, and Industrial Park) and 5 county-level cities. $\mathrm{SCH}$, which is in Gusu, is the only tertiary children's hospital in Suzhou. In 2016, the hospital recorded $\approx 1.9$ million outpatient and emergency visits and 45,000 hospitalizations. Hospital records indicate that, during $2011-2014$, a total of $84.9 \%$ of patients $<5$ years of age with influenza-like illness and $63.3 \%$ of patients $<5$ years of age with meningitis or encephalitis resided in the 5 municipal districts of Suzhou (12). According to a 2011 healthcare utilization survey $(13,14)$, SCH patients accounted for $67.7 \%$ of total discharges of children $<5$ years of age from all 96 hospitals in downtown Suzhou. We defined the catchment area of SCH as the 5 municipal districts of Suzhou; we assumed that $67.7 \%$ of children $<5$ years of age who resided in the catchment areas would be treated at $\mathrm{SCH}$. The study was conducted in accordance with internationally recognized standards for ethical research and was approved by the institutional review boards of the School of Public Health, Fudan University (approval no. 2015-04-0545) and SCH.

\section{Case Definition, Identification, and Serotyping}

We defined IPD patients as those from whom S. pneumoniae was isolated from a normally sterile body site such as cerebrospinal fluid (CSF), blood, or pleural fluid. Within 24 hours after admission, the hospital collects CSF specimens from patients with suspected meningitis, defined as acute onset of fever with change in mental status, meningeal signs (e.g., neck stiffness, headache), or both; blood cultures from patients with a temperature of $>39.0^{\circ} \mathrm{C}$; and pleural fluid from patients with suspected effusion detected in chest radiography or computed tomography scan. The specimens were sent to the laboratory at $\mathrm{SCH}$ for bacterial culture $\leq 2$ hours after collection according to the standard methods of collection, transport, and culturing (7). Laboratory physicians identified isolates as S. pneumoniae by morphologic features, a-hemolysis, Gram staining, and bile solubility or optochin susceptibility using Oxoid Optochin Discs (Thermo Fisher Scientific, https://www.thermofisher.com).

We collected the S. pneumoniae-positive isolates from $\mathrm{SCH}$ and sent them to the Key Laboratory of Public Health Safety at Fudan University, Shanghai, for serotyping. We identified the serotypes of pneumococcal isolates using the Quellung reaction with antisera (Statens Serum Institute, https://en.ssi.dk), a multiplex PCR described previously (15), or both.

\section{Demographic Information}

We obtained information on the annual population of children $<5$ years of age in the catchment area during 2010-2017 from the immunization program database at the Suzhou Center for Disease Control and Prevention, which serves all residents and $>95 \%$ of the migrant population in this age group in Suzhou. Almost all newborn children in Suzhou, regardless of residence, are immunized; all immunizations are logged in the program database.

\section{Estimating Incidence of Hospitalization for IPD}

We used a rapid method from the World Health Organization to calculate the incidence of hospitalization for IPD (11). This method uses the sentinel hospital's meningitis surveillance system to identify the numerator as potential bacterial meningitis cases and the denominator as the estimated number of children at risk for meningitis in the catchment area. China does not have a nationwide meningitis surveillance system, but $\mathrm{SCH}$ collects routine CSF, blood, and pleural fluid cultures from children with suspected IPD. We estimated the denominator (i.e., children at risk for IPD in the area) by multiplying the population $<5$ years of age in the catchment area by the percentage of children in that age group who are treated at SCH $(67.7 \%)$. Thus, we calculated the incidence of hospitalization for IPD in the catchment area of $\mathrm{SCH}(\mathrm{Y})$ as

$$
Y=\frac{\text { IPD cases in } \mathrm{SCH}}{\text { Children }<5 \text { years of age in catchment area } \times 67.7 \%}
$$

We used the Wilson method for binominal distribution to estimate 95\% CIs of IPD hospitalizations. We used the $\chi^{2}$ test to compare the incidence of hospitalization across different age groups. We conducted all statistical analyses using SAS version 9.4 (SAS Institute, https:/ / www.sas.com).

\section{Results}

\section{Characteristics of IPD Patients}

During January 2010-December 2017, SCH collected 20,260 CSF specimens from children $<5$ years of age. Among the specimens, 283 tested positive for bacterial infections, including $46(16.3 \%)$ positive for $S$. pneumoniae and $51(18.0 \%)$ positive for Staphylococcus epidermidis. SCH also collected 62,858 blood and 
138 pleural effusion specimens for bacterial detection from children $<5$ years of age. Of these samples, 2,432 blood and 29 pleural effusion cultures tested positive for bacterial infection. S. pneumoniae was the sixth most common bacteria in the positive blood cultures $(110 / 2,432 ; 4.5 \%)$ and the most common bacteria in the positive pleural effusion cultures $(16 / 29 ; 55.2 \%)$. The proportion of samples that tested positive for $S$. pneumoniae from children $<5$ years of age were $0.2 \%(46 / 20,260)$ in CSF, $0.2 \%$ $(110 / 62,858)$ in blood, and $11.6 \%(16 / 138)$ in pleural effusion samples. After accounting for duplicate specimens, $\mathrm{SCH}$ identified 105 patients $<5$ years of age with IPD during 2010-2017. The male:female ratio was $1.3 ; 54.3 \%$ were $2-<5$ years of age. Common diagnoses included meningitis $(31.4 \%)$, pneumonia $(28.6 \%)$, and sepsis $(21.0 \%)$ (Table 1$)$.

\section{Incidence of Hospitalization for Children with IPD}

The population of children $<5$ years of age in the catchment area increased from 90,756 in 2010 to 171,676 in 2017. During January 2010-December 2017, the estimated annual incidence of hospitalization for IPD among children in this age group in Suzhou ranged from 8.16 to 17.86 per 100,000 children, peaking in 2015 (Table 2). The incidence of hospitalization for IPD fluctuated without significance $\left(\chi^{2}=\right.$ $1.51 ; p>0.05$ ) (Figure 1). The IPD hospitalization incidence differed among age groups and was highest among children $<1$ year of age $\left(x^{2}=6.73 ; p<0.05\right)$. The incidence of hospitalization among children $<5$ years of age was 4.57 (95\% CI 3.26-6.42)/100,000 children for meningitis, 4.16 (95\% CI 2.91-5.93)/100,000 children for bacteremic pneumonia, and 3.05 (95\% CI 2.01-4.62)/100,000 children for sepsis.

\section{Serotype Distribution and Incidence of IPD Hospitalizations by Serotype}

SCH sent 74 (70.5\%) pneumococcal isolates from patients with IPD to the laboratory for serotyping. We identified 8 different capsular serotypes: 6B (21/74; $28.4 \%), 14(14 / 74 ; 18.9 \%), 19 \mathrm{~A}(14 / 74 ; 18.9 \%), 19 \mathrm{~F}$ (9/74; 12.2\%), 23F (8/74; 10.8\%), $20(3 / 74 ; 4.1 \%), 9 \mathrm{~V}$ $(3 / 74 ; 4.1 \%)$, and $15 \mathrm{~B} / \mathrm{C}(2 / 74 ; 2.7 \%)$. Thus, the serotype coverage rate, or the percentage of cases caused by serotypes preventable by vaccination, of PCV10 was $74.4 \%$ and PCV13 was $93.2 \%$.

Among patients with meningitis, serotypes $6 \mathrm{~B}$ $(10 / 25 ; 40.0 \%)$ and $14(6 / 25 ; 24.0 \%)$ were most common. Of patients with sepsis, the most common serotypes were $23 \mathrm{~F}(5 / 14 ; 35.7 \%)$ and 19A (3/14; $21.4 \%)$ (Figure 2). However, these findings were not significant. We calculated the estimated serotype-specific IPD hospitalization incidences to be 4.13 (95\% CI 2.89-5.90)/100,000 children for $6 \mathrm{~B}, 2.75$ (95\% CI 1.78-4.26)/100,000 children for 14, 2.75 (95\% CI 1.78-4.26)/100,000 children for 19A, 1.77 (95\% CI 1.03-3.04)/100,000 children for 19F, 1.57 (95\% CI 0.89-2.89)/100,000 children for 23F, 0.59 (95\% CI $0.24-$ 1.48)/100,000 children for 20 and $9 \mathrm{~V}$, and 0.39 (95\% CI 0.13-1.19)/100,000 children for 15B/C (Figure 2).

\section{Discussion}

We describe children hospitalized for IPD at SCH during January 2010-December 2017. We used

\begin{tabular}{|c|c|c|c|c|}
\hline \multirow[b]{2}{*}{ Characteristic } & \multicolumn{4}{|c|}{ Age group, no. (\%) } \\
\hline & $<1 \mathrm{y}$ & $1-<2 y$ & $2-<5 y$ & Total \\
\hline Total & $31(100.0)$ & $17(100.0)$ & $57(100.0)$ & $105(100.0)$ \\
\hline \multicolumn{5}{|l|}{ Sex } \\
\hline M & $17(54.8)$ & $10(58.8)$ & 33 (57.9) & $60(57.1)$ \\
\hline $\mathrm{F}$ & $14(45.2)$ & $7(41.2)$ & $24(42.1)$ & $45(42.9)$ \\
\hline \multicolumn{5}{|l|}{ Year } \\
\hline 2010 & $5(16.1)$ & $1(5.9)$ & $4(7.0)$ & $10(9.5)$ \\
\hline 2011 & $4(12.9)$ & $4(23.5)$ & $3(5.3)$ & $11(10.5)$ \\
\hline 2012 & $4(12.9)$ & $1(5.9)$ & $5(8.8)$ & $10(9.5)$ \\
\hline 2013 & $5(16.1)$ & $4(23.5)$ & $4(7.0)$ & $13(12.4)$ \\
\hline 2014 & $4(12.9)$ & $4(23.5)$ & $9(15.8)$ & $17(16.2)$ \\
\hline 2015 & $5(16.1)$ & $1(5.9)$ & $12(21.1)$ & $18(17.1)$ \\
\hline 2016 & $2(6.5)$ & $1(5.9)$ & $6(10.5)$ & $9(8.6)$ \\
\hline 2017 & $2(6.5)$ & $1(5.9)$ & $14(24.6)$ & $17(16.2)$ \\
\hline \multicolumn{5}{|l|}{ Primary discharge diagnosis } \\
\hline Meningitis & $16(51.6)$ & $4(23.5)$ & $13(22.8)$ & $33(31.4)$ \\
\hline Pneumonia & $7(22.6)$ & $3(17.6)$ & $20(35.1)$ & 30 (28.6) \\
\hline Sepsis & $6(19.4)$ & $6(35.3)$ & $10(17.5)$ & $22(21.0)$ \\
\hline Upper respiratory infection & $1(3.2)$ & $1(5.9)$ & $1(1.8)$ & $3(2.9)$ \\
\hline Bronchitis & 0 & $1(5.9)$ & $1(1.8)$ & $2(1.9)$ \\
\hline Other* & $1(3.2)$ & $2(11.8)$ & $12(21.1)$ & $15(14.3)$ \\
\hline
\end{tabular}

*Other conditions: leukemia (6 cases), anemia (1 case), endocarditis (2 cases), arrhythmia (2 cases), acute otitis media (1 case), jaundice (1 case), acute gastritis (1 case), and psoas abscess (1 case). 
Table 2. Estimated IPD hospitalization incidence among children $<5$ years of age, Suzhou, China, 2010-2017*

\begin{tabular}{|c|c|c|c|c|c|c|c|c|c|}
\hline \multirow[b]{2}{*}{ Year } & \multicolumn{3}{|c|}{ Children $<1$ y of age } & \multicolumn{3}{|c|}{ Children $1-<2$ y of age } & \multicolumn{3}{|c|}{ Children $2-<5$ y of age } \\
\hline & $\begin{array}{l}\text { IPD cases } \\
\text { at SCH }\end{array}$ & Pop.† & $\mathrm{HI} \neq(95 \% \mathrm{Cl})$ & $\begin{array}{l}\text { IPD cases } \\
\text { at } \mathrm{SCH}\end{array}$ & Pop.† & $\mathrm{HI} \ddagger(95 \% \mathrm{Cl})$ & $\begin{array}{l}\text { IPD cases } \\
\text { at } \mathrm{SCH}\end{array}$ & Pop.† & HI‡ $(95 \%$ Cl) \\
\hline 2010 & 5 & 21,510 & $\begin{array}{c}34.33 \\
(14.67-80.35)\end{array}$ & 1 & 19,433 & $\begin{array}{c}7.60 \\
(1.34-43.02)\end{array}$ & 4 & 49,813 & $\begin{array}{c}11.86 \\
(4.61-30.51)\end{array}$ \\
\hline 2011 & 4 & 22,731 & $\begin{array}{c}25.99 \\
(10.11-66.81)\end{array}$ & 4 & 20,136 & $\begin{array}{c}29.34 \\
(11.41-75.43)\end{array}$ & 3 & 50,527 & $\begin{array}{c}8.77 \\
(2.98-25.79)\end{array}$ \\
\hline 2012 & 4 & 26,452 & $\begin{array}{c}22.35 \\
(8.69-57.46)\end{array}$ & 1 & 25,483 & $\begin{array}{c}5.80 \\
(1.02-32.83)\end{array}$ & 5 & 70,423 & $\begin{array}{c}10.49 \\
(4.48-25.54)\end{array}$ \\
\hline 2013 & 5 & 23,893 & $\begin{array}{c}30.91 \\
(13.20-72.35)\end{array}$ & 4 & 31,228 & $\begin{array}{c}18.92 \\
(7.36-48.65)\end{array}$ & 4 & 76,041 & $\begin{array}{c}7.77 \\
(3.02-19.98)\end{array}$ \\
\hline 2014 & 4 & 29,110 & $\begin{array}{c}20.30 \\
(7.89-52.18)\end{array}$ & 4 & 28,006 & $\begin{array}{c}21.10 \\
(8.21-54.26)\end{array}$ & 9 & 87,752 & $\begin{array}{c}15.15 \\
(7.97-28.79)\end{array}$ \\
\hline 2015 & 5 & 22,745 & $\begin{array}{c}32.48 \\
(13.87-76.02)\end{array}$ & 1 & 34,119 & $\begin{array}{c}4.33 \\
(0.76-24.53)\end{array}$ & 12 & 92,038 & $\begin{array}{c}19.26 \\
(11.02-33.66)\end{array}$ \\
\hline 2016 & 2 & 32,360 & $\begin{array}{c}9.13 \\
(2.5-33.28)\end{array}$ & 1 & 27,768 & $\begin{array}{c}5.32 \\
(0.94-30.13)\end{array}$ & 6 & 102,818 & $\begin{array}{c}8.62 \\
(3.95-18.80)\end{array}$ \\
\hline 2017 & 2 & 31,415 & $\begin{array}{c}9.40 \\
(2.58-34.28)\end{array}$ & 1 & 38,255 & $\begin{array}{c}3.86 \\
(0.68-21.88) \\
\end{array}$ & 14 & 102,006 & $\begin{array}{c}20.27 \\
(12.07-34.02) \\
\end{array}$ \\
\hline Total & 31 & 210,216 & $\begin{array}{c}21.78 \\
(15.34-30.91)\end{array}$ & 17 & 224,428 & $\begin{array}{c}11.19 \\
(6.99-17.92)\end{array}$ & 57 & 631,418 & $\begin{array}{c}13.33 \\
(10.29-17.27)\end{array}$ \\
\hline
\end{tabular}

previous studies (12-14) to estimate the incidence of hospitalization for IPD among children $<5$ years of age in Suzhou. During January 2010-December 2017, the annual incidence of hospitalization for IPD in Suzhou ranged from 8.16 to $17.86 / 100,000$ children and the average incidence was $14.55 / 100,000$ children. The annual incidence of IPD did not significantly change during the 8-year period, peaking at 17.86/100,000 children in 2015. The IPD hospitalization incidence was highest $(21.78$ hospitalizations $/ 100,000$ children) among those $<1$ year of age and lowest (13.33 hospitalizations/100,000 children) among those $2-<5$ years of age.

We estimated the incidence of hospitalization for pneumococcal meningitis as $4.57 / 100,000$ children. A systematic review in China estimated the mean incidence of pneumococcal meningitis in children 1-23 months of age as 5.1 cases/100,000 children during
1999-2003 (16). Another study in China estimated the incidence of bacterial meningitis as 6.95-22.30 cases/100,000 children $<5$ years of age during 20062009; S. pneumoniae caused $53 \%$ of these cases (10), indicating a pneumococcal meningitis incidence of $\approx 3.7-11.8 / 100,000$ children. This incidence is comparable to our estimates. A study in Beijing estimated the incidence of sepsis in adults as 461 cases/100,000 persons (17), but that study did not classify patients on the basis of bacterial infection. Many studies around the world indicate similar incidences of IPD before the use of PCVs (18-20). A 20-year study in Atlanta, Georgia, USA, showed that during 2003-2013, the overall IPD rate was 8.03-14.02 cases/100,000 persons; incidence was also estimated by using $S$. pneumoniae isolates collected from normally sterile sites (4). The IPD incidence of children $<5$ years of age
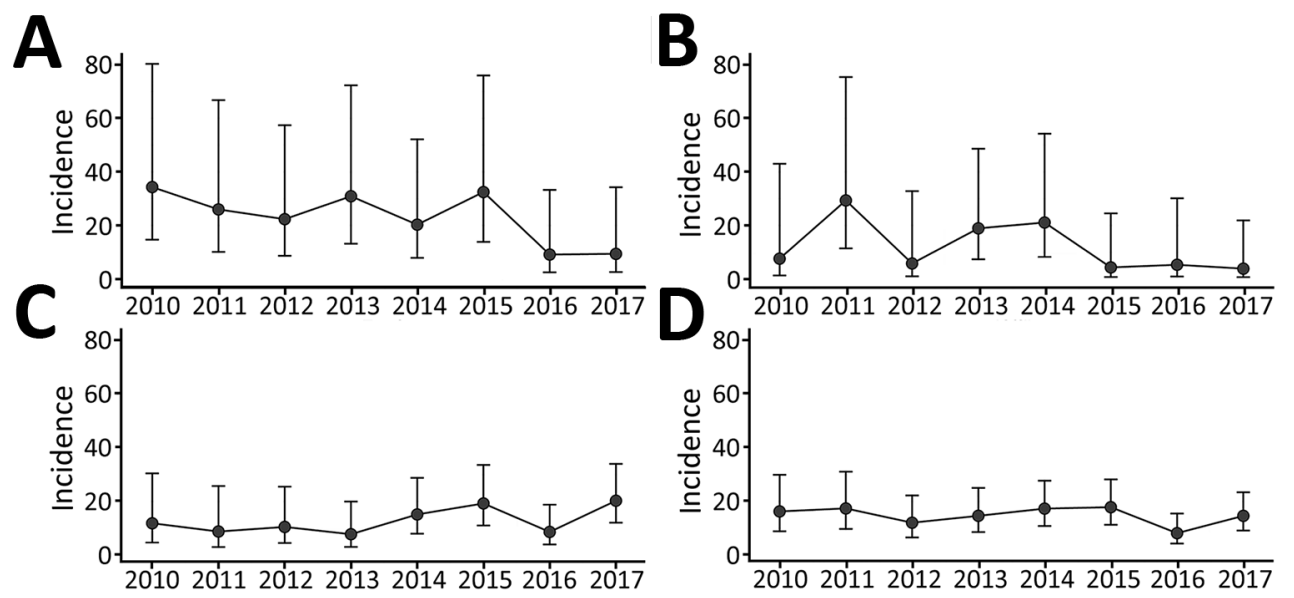

Figure 1. Estimated invasive pneumococcal diseases hospitalization incidence per 100,000 children among children $<5$ years of age, by age and year, Suzhou, China, 2010-2017. A) Children <1 year of age; B) children 1-<2 years of age; $C$ ) children $2-<5$ years of age; D) children $<5$ years of age. Error bars indicate $95 \% \mathrm{Cls}$. 


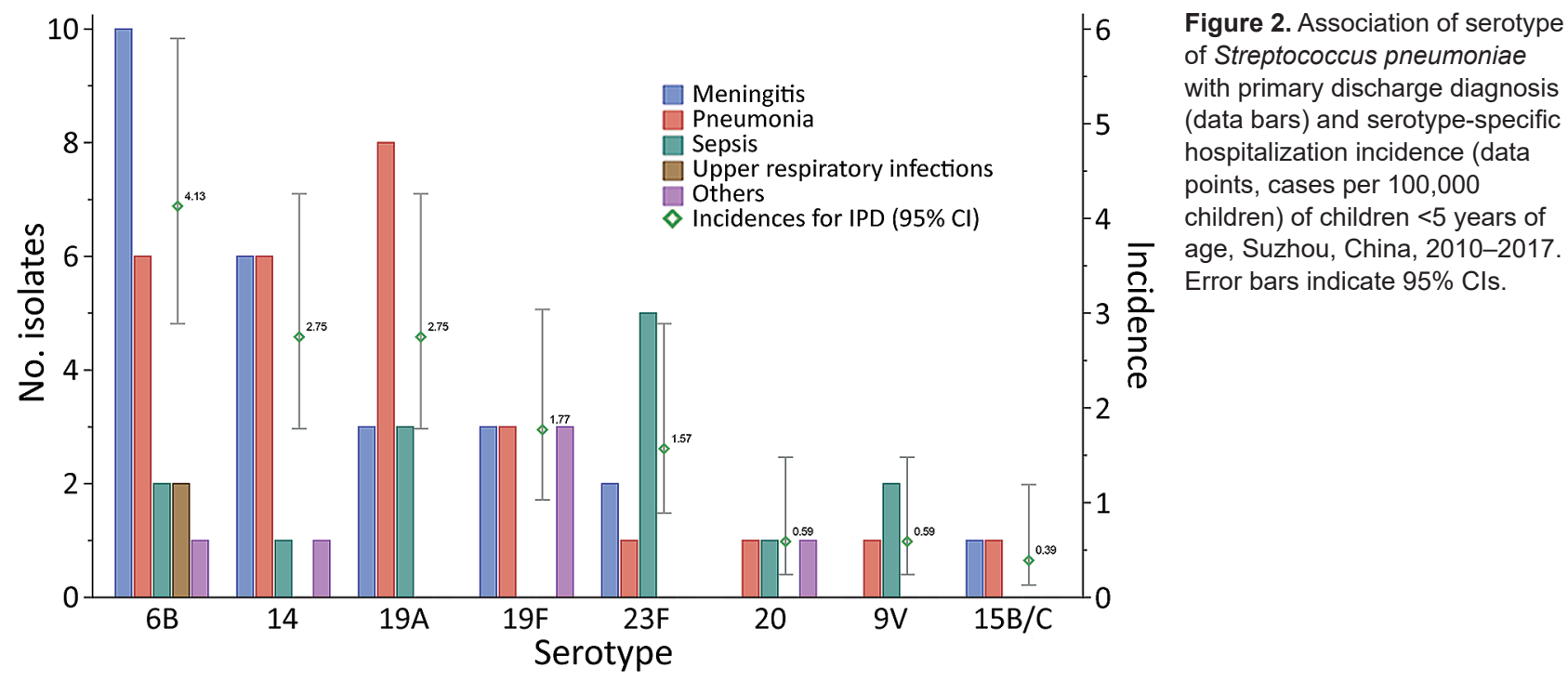

in India in 2006 was 1,500 cases/100,000 persons (5), much higher than in China and other countries.

The proportion of CSF, blood, and pleural effusion samples that tested positive for S. pneumoniae were lower than those found in studies in India and Turkey $(21,22)$. Possible reasons for the lower proportion of $S$. pneumoniae-positive samples included the common use of antimicrobial drugs before hospital admission $(7,10)$ and the use of only traditional bacterial cultures, which are less sensitive than immunochromatography and PCR, for identification of S. pneumoniae. We did not collect data on antimicrobial drug usage, but our previous study in the same hospital during 2006-2007 found that $\approx 86 \%$ of children with lower respiratory tract infections used antimicrobial drugs, not all of which were prescribed, before admission (23). Li et al. showed that, in China's general hospitals, the percentage of outpatients treated with prescribed antimicrobial drugs declined from $20.17 \%$ in 2012 to $12.94 \%$ in 2016 (24). Since 2012, China has improved the judicious use of antimicrobial drugs; however, the inappropriate use of antimicrobial drugs in children remains a serious problem $(25,26)$. We might underestimate the incidence of hospitalization for IPD because pretreating with antimicrobial drugs at home might reduce the proportion of IPD patients who test positive for S. pneumoniae.

In this study, we found that serotypes $6 \mathrm{~B}, 14$, and 19A caused $66.2 \%$ of all IPD infections. These results differ from those of our recent systematic review (27), which found 19F and 19A to be the most common serotypes among invasive strains isolated from children in mainland China during 2000-2016. Furthermore, a study in England and Wales found serotype 18C to be the most common serotype among children $<5$ years of age with meningitis (28). Therefore, we believe the distribution of pneumococcal serotypes varies with geographic location.

PCVs have been introduced in $>100$ countries, possibly contributing to a major reduction in the incidence of IPD (29). During most of the study period, PCV7 was the only PCV available in mainland China; whether this vaccination decreased the incidence of IPD in China is unknown. PCV7 uptake in China was very low, an estimated $2 \%-7 \%(8,9)$. Our previous study showed that $87.4 \%$ of IPD cases among children in mainland China (27) were caused by pneumococcal serotypes preventable by PCV13; in the study we report here, that proportion increased to $93.2 \%$. Therefore, if the vaccine uptake of PCV13 in China is sufficiently high, we expect similar reductions in the incidence of IPD in children in China, as has been observed in other countries (29).

The major limitation of this study is that common antimicrobial drug use might have reduced the proportion of specimens that tested positive for S. pneumoniae, causing us to underestimate the IPD hospitalization incidence. In addition, $\mathrm{SCH}$ only took blood cultures of patients with a temperature $>39.0^{\circ} \mathrm{C}$; this policy might overlook IPD patients without fever. Furthermore, we did not have data on IPD patients in Suzhou who were not treated at SCH. Because SCH treated $67.7 \%$ of children $<5$ years of age who were discharged from hospitals in downtown Suzhou in 2011 (13), we used this proportion to estimate the number of children at risk for IPD in Suzhou; we might have overestimated or underestimated the incidence of IPD. Our previous study found that $\approx 50 \%$ of children with influenza-like illness in the catchment area are treated atSCH (12). BecauseSCH is the only pediatric tertiary hospital in Suzhou and IPDs are more severe than influenza-like illnesses, we assumed that the 
proportion of children with IPD in the catchment area who were treated at $\mathrm{SCH}$ would be $>50 \%$. Finally, the small number of identified IPD cases might skew our estimates.

In conclusion, we found that the incidence of hospitalization for IPD in Suzhou was similar to that of populations in China and other countries before the use of PCVs. In addition, the IPD hospitalization incidence did not significantly change during 2010-2017. In China, a high proportion of pneumococcal serotypes are preventable with PCV13. These results provide baseline data of IPD incidence before PCV13 use in China.

\section{Acknowledgements}

We thank the physicians and the laboratory staff at Soochow University Affiliated Children Hospital who contributed to this study. We also thank Zhang Fangmin for assistance with data collection.

This study was supported by the National Natural Science Foundation of China (grant no. 81102166), the Shanghai Municipal Health and Family Planning (grant nos. GWTD2015S05 and 15GWZK0101), the National Key Research and Development Program of China (grant no. 2017YFC0211700), the SINO-US collaborative program on Emerging and Re-Emerging Infectious Diseases (grant no. 5U2GGH000018), and the Application Research on Key Technologies of People's Livelihood Science and Technology in Suzhou (grant no. SS201867).

\section{About the Author}

Ms. Chen has an MPH from Fudan University. Her research interests include the epidemiology of infectious diseases and pneumococcal diseases.

\section{References}

1. Weinberger DM, Harboe ZB, Sanders EAM, Ndiritu M, Klugman KP, Rückinger S, et al. Association of serotype with risk of death due to pneumococcal pneumonia: a meta-analysis. Clin Infect Dis. 2010;51:692-9. https://doi.org/10.1086/655828

2. World Health Organization. Pneumococcal conjugate vaccine for childhood immunization. 2007 [cited 2018 Jun 30]. https://apps.who.int/iris/handle/10665/240901

3. O'Brien KL, Wolfson LJ, Watt JP, Henkle E, Deloria-Knoll M, McCall N, et al.; Hib and Pneumococcal Global Burden of Disease Study Team. Burden of disease caused by Streptococcus pneumoniae in children younger than 5 years: global estimates. Lancet. 2009;374:893-902. https:/ / doi.org/ 10.1016/S0140-6736(09)61204-6

4. Schroeder MR, Chancey ST, Thomas S, Kuo WH, Satola SW, Farley MM, et al. A population-based assessment of the impact of 7- and 13-valent pneumococcal conjugate vaccines on macrolide-resistant invasive pneumococcal disease: emergence and decline of Streptococcus pneumoniae serotype
19A (CC320) with dual macrolide resistance mechanisms. Clin Infect Dis. 2017;65:990-8. https://doi.org/10.1093/ cid/cix446

5. Shah AS, Nisarga R, Ravi Kumar KL, Hubler R, Herrera G, Kilgore PE. Establishment of population-based surveillance for invasive pneumococcal disease in Bangalore, India. Indian J Med Sci. 2009;63:498-507. https:/ / doi.org/10.4103/ 0019-5359.58879

6. Kang CI, Song JH. Antimicrobial resistance in Asia: current epidemiology and clinical implications. Infect Chemother. 2013;45:22-31. https:// doi.org/10.3947/ic.2013.45.1.22

7. Zhang X, Tian J, Shan W, Xue J, Tao Y, Geng Q, et al. Characteristics of pediatric invasive pneumococcal diseases and the pneumococcal isolates in Suzhou, China before introduction of PCV13. Vaccine. 2017;35:4119-25. https://doi.org/10.1016/j.vaccine.2017.06.046

8. Mo X, Tobe RG, Liu X, Mori R. Cost-effectiveness and health benefits of pediatric 23-valent pneumococcal polysaccharide vaccine, 7 -valent pneumococcal conjugate vaccine and forecasting 13-valent pneumococcal conjugate vaccine in China. Pediatr Infect Dis J. 2016;35:e353-61. https://doi.org/10.1097/INF.0000000000001288

9. Boulton ML, Ravi NS, Sun X, Huang Z, Wagner AL. Trends in childhood pneumococcal vaccine coverage in Shanghai, China, 2005-2011: a retrospective cohort study. BMC Public Health. 2016;16:109. https:/ / doi.org/10.1186/ s12889-016-2785-7

10. Li Y, Yin Z, Shao Z, Li M, Liang X, Sandhu HS, et al.; Acute Meningitis and Encephalitis Syndrome Study Group. Population-based surveillance for bacterial meningitis in China, September 2006-December 2009. Emerg Infect Dis. 2014;20:61-9. https:/ / doi.org/10.3201/ eid2001.120375

11. Home C; Centers for Disease Control and Prevention (CDC). Estimating meningitis hospitalization rates for sentinel hospitals conducting invasive bacterial vaccine-preventable diseases surveillance. MMWR Morb Mortal Wkly Rep. 2013;62:810-2.

12. Zhang $\mathrm{T}$, Zhang J, Hua J, Wang D, Chen L, Ding Y, et al. Influenza-associated outpatient visits among children less than 5 years of age in eastern China, 2011-2014. BMC Infect Dis. 2016;16:267. https:// doi.org/10.1186/s12879-016-1614-Z

13. Zhang X, Zhang J, Chen L, Feng L, Yu H, Zhao G, et al. Pneumonia and influenza hospitalizations among children under 5 years of age in Suzhou, China, 2005-2011. Influenza Other Respir Viruses. 2017;11:15-22. https://doi.org/10.1111/irv.12405

14. Shan W, Shi T, Zhang X, Xue J, Wang Y, Yu J, et al. Hospitalization rate and population-based incidence of hospitalization for community-acquired pneumonia among children in Suzhou, China. Pediatr Infect Dis J. 2018;37:1242-7.

15. Geng Q, Zhang T, Ding Y, Tao Y, Lin Y, Wang Y, et al. Molecular characterization and antimicrobial susceptibility of Streptococcus pneumoniae isolated from children hospitalized with respiratory infections in Suzhou, China. PLoS One. 2014;9:e93752. https://doi.org/10.1371/ journal.pone.0093752

16. Lin TY, Shah NK, Brooks D, Garcia CS. Summary of invasive pneumococcal disease burden among children in the Asia-Pacific region. Vaccine. 2010;28:7589-605. https://doi.org/10.1016/j.vaccine.2010.07.053

17. Zhou J, Tian H, Du X, Xi X, An Y, Duan M, et al.; for China Critical Care Clinical Trials Group (CCCCTG). Population-based epidemiology of sepsis in a subdistrict of Beijing. Crit Care Med. 2017;45:1168-76. https:/ / doi.org/ 10.1097/CCM.0000000000002414 
18. Rendi-Wagner P, Georgopoulos A, Kundi M, Mutz I, Mattauch M, Nowak J, et al. Prospective surveillance of incidence, serotypes and antimicrobial susceptibility of invasive Streptococcus pneumoniae among hospitalized children in Austria. J Antimicrob Chemother. 2004;53:826-31. https://doi.org/10.1093/jac/dkh211

19. von Kries R, Hermann M, Hachmeister A, Siedler A, Schmitt HJ, Al-Lahham A, et al. Prediction of the potential benefit of different pneumococcal conjugate vaccines on invasive pneumococcal disease in German children. Pediatr Infect Dis J. 2002;21:1017-23. https:/ / doi.org/ 10.1097/00006454-200211000-00008

20. von Kries, Siedler A, Schmitt HJ, Reinert RR. Proportion of invasive pneumococcal infections in German children preventable by pneumococcal conjugate vaccines. Clin Infect Dis. 2000;31:482-7.

21. Manoharan A, Manchanda V, Balasubramanian S, Lalwani S, Modak M, Bai S, et al.; Alliance for Surveillance of Invasive Pneumococci Study Group. Invasive pneumococcal disease in children aged younger than 5 years in India: a surveillance study. Lancet Infect Dis. 2017;17:305-12. https:/ / doi.org/10.1016/S1473-3099(16)30466-2

22. Ceyhan M, Yildirim I, Balmer P, Borrow R, Dikici B, Turgut $\mathrm{M}$, et al. A prospective study of etiology of childhood acute bacterial meningitis, Turkey. Emerg Infect Dis. 2008;14:1089-96. https://doi.org/10.3201/ eid1407.070938

23. Zhang T, Black S, Hao C, Ding Y, Ji W, Chen R, et al. The blind nasotracheal aspiration method is not a useful tool for pathogen detection of pneumonia in children. PLoS One. 2010;5:e15885. https:// doi.org/10.1371/journal. pone. 0015885

24. Li D. Trend analysis of antibiotic use in China's general hospitals during 2012-2016 [master's thesis]. CNKI.net. 2019 [cited 2019 Jul 1]. https:/ / tinyurl.com/trendanalysisli2019

25. Chang J, Xu S, Zhu S, Li Z, Yu J, Zhang Y, et al. Assessment of non-prescription antibiotic dispensing at community pharmacies in China with simulated clients: a mixed cross-sectional and longitudinal study. Lancet Infect Dis. 2019;19:1345-54. https:/ / doi.org/10.1016/ S1473-3099(19)30324-X

26. Measures for the administration of clinical application of antibiotics (Order No. 84 of the Ministry of Health) [in Chinese]. 2012 [cited 2018 Jun 10]. http:/ / www.nhc.gov.cn/ fzs/s3576/201205/2f773c2ddbd84e19aab0b4b2d9741900. shtml

27. Chen K, Zhang X, Shan W, Zhao G, Zhang T. Serotype distribution of Streptococcus pneumoniae and potential impact of pneumococcal conjugate vaccines in China: A systematic review and meta-analysis. Hum Vaccin Immunother. 2018;14:453-463.

28. van Hoek AJ, Andrews N, Waight PA, George R, Miller E. Effect of serotype on focus and mortality of invasive pneumococcal disease: coverage of different vaccines and insight into non-vaccine serotypes. PLoS One. 2012;7:e39150. https://doi.org/10.1371/journal.pone.0039150

29. Rodgers GL, Klugman KP. The future of pneumococcal disease prevention. Vaccine. 2011;29:C43-8. https:/ / doi.org/ 10.1016/j.vaccine.2011.07.047

Address for correspondence: Tao Zhang, Department of Epidemiology, School of Public Health, Fudan University, PO Box 289, 138 Yi Xue Yuan Rd, Shanghai 200032, China; email: tzhang@ shmu.edu.cn; Xuejun Shao, Soochow University Affiliated Children's Hospital, Suzhou 215008, China; email: xjshao@suda.edu.cn

\section{EID Podcast} Developing Biological Reference Materials to Prepare for Epidemics

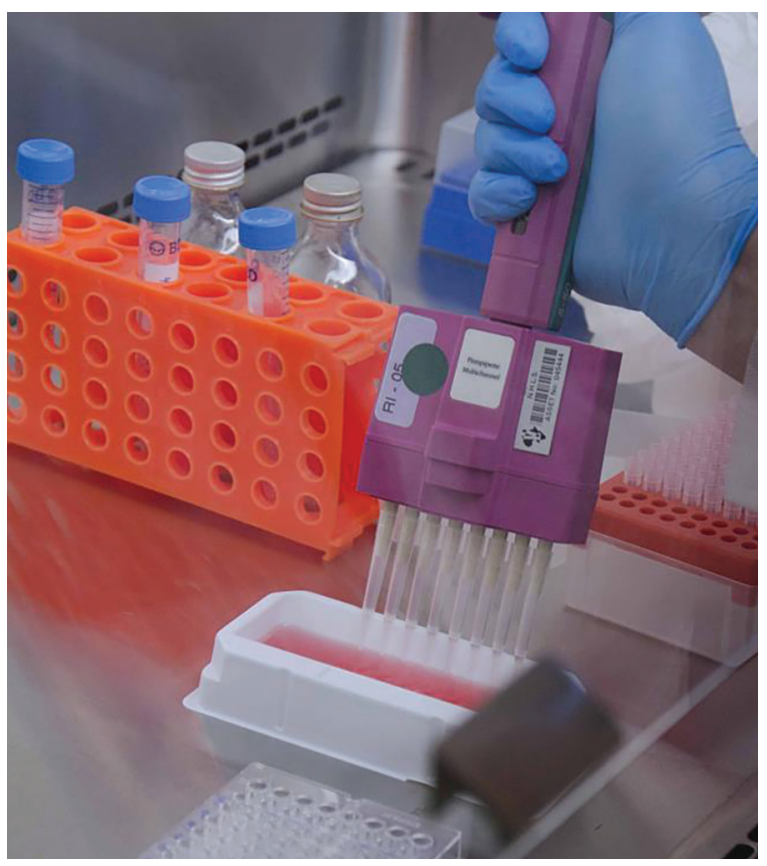

Having standard biological reference materials, such as antigens and antibodies, is crucial for developing comparable research across international institutions. However, the process of developing a standard can be long and difficult.

In this EID podcast, Dr. Tommy Rampling, a clinician and academic fellow at the Hospital for Tropical Diseases and University College in London, explains the intricacies behind the development and distribution of biological reference materials.

\section{Visit our website to listen:} https: //go.usa.gov/xyfJX

\section{EMERGING}

INFECTIOUS DISEASES 